\title{
The shortest period M dwarf eclipsing system BW3 V38
}

\section{Determination of absolute elements ${ }^{\star}$}

\author{
C. Maceroni ${ }^{1}$ and J. Montalbán ${ }^{2}$ \\ 1 INAF - Osservatorio Astronomico di Roma via Frascati 33, 00040 Monteporzio C. (RM), Italy \\ e-mail: maceroni@mporzio.astro.it \\ 2 Institut d'Astrophysique et Géophysique, Université de Liège, Allée du 6 Août, 4000 Liège, Belgium \\ e-mail: j.montalban@ulg.ac.be
}

Received 29 March 2004 / Accepted 22 June 2004

\begin{abstract}
The spectroscopic data for the short-period (0.1984) eclipsing binary V38, discovered by the OGLE micro-lensing team in Baade's Window field BW3, are analyzed. Radial velocity curves are derived from mid-resolution spectra obtained with EMMI-NTT at ESO - La Silla, and a simultaneous solution of the existing light curve by OGLE and of the new radial velocity curves is obtained. The system is formed by almost twin M3e dwarf components that are very close, but not yet in contact. The spectra of both dwarfs show signatures of the presence of strong chromospheres. Spectroscopy definitely confirms, therefore, what was suggested on the basis of photometry: BW3 V38 is indeed a unique system, as no other similar binary with $\mathrm{M}$ components and in such a tight orbit is known.

Within the limits posed by the relatively large errors, due to the combined effect of system faintness and of the constraints on exposure time, the derived physical parameters seem to agree with the relations obtained from the other few known eclipsing binaries with late type components (which indicate a discrepancy between the available evolutionary models and the data at $\sim 10 \%$ level). A possible explanation is the presence of strong magnetic fields and fast rotation (that applies to the BW3 V38 case as well). A simple computation of the system secular evolution by angular momentum loss and spin-orbit synchronization shows that the evolution of a system with $\mathrm{M}$ dwarf components is rather slow, and indicates as well a possible reason why systems similar to BW3 V38 are so rare.
\end{abstract}

Key words. stars: binaries: close - stars: binaries: eclipsing - stars: late-type - stars: fundamental parameters stars: individuals: OGLE BW3 V38

\section{Introduction}

OGLE BW03 V038 (BW3 V38, for brevity, through this paper; $\alpha_{2000}=18^{\mathrm{h}} 04^{\mathrm{m}} 44^{\mathrm{s}} .19, \delta_{2000}=-30^{\circ} 09^{\prime} 05^{\prime \prime}, P=0.19839$, $\left.I_{\max }=15.83,(V-I)_{\max }=2.45, \Delta I=0.78\right)$ appears in the second instalment of the OGLE Catalog of periodically variable stars (Udalski et al. 1995) and is therein classified, on the basis of light curve shape, as a contact binary of W UMa-type $(E W)$. It was later rejected, however, from the bona-fide sample of contact systems in the OGLE fields, which was selected by Rucinski (1997) by means of an objective Fourier-based classification of the light curves. The system was found marginally outside the region that, in the space of parameters defining the light curve properties, corresponds to the contact configuration.

Maceroni \& Rucinski (1997, hereafter Paper I) analyzed the OGLE photometric data, providing a solution of the $I$-band light curve and studying in detail the related uncertainties. They reported, as well, that the system is undetected in the

^ Based on observations collected at the European Southern Observatory, La Silla, Chile.
ROSAT survey, with an upper limit to the X-ray flux of $1.8 \times$ $10^{-13} \mathrm{erg} \mathrm{cm}^{-2} \mathrm{~s}^{-1}$ (this result was not surprising on the basis of the system properties and of a rough estimate of its distance of $\sim 400 \mathrm{pc}$ ).

The de-reddened $V-I$ color of BW3 V38 $\left((V-I)_{0}=\right.$ 2.3-2.4, Paper I), its light curve solution and the short period suggest a configuration with two similar very close and fast rotating M-dwarfs. This makes of BW3 V38 an extremely interesting object on several grounds.

While a few eclipsing binaries with M-type components are known at present (see the recent paper by Ribas 2003, for a summary) BW3 V38 is the only one with so tight an orbit, and this, according to the current knowledge on close binary formation, is very unlikely to be its original configuration, as "in situ" formation is ruled out for such small separations (see Sect. 4).

We think, therefore, that BW3 V38 started its MS life with a larger separation and has evolved through the combined effect of angular momentum loss by magnetic braking (AML) and of spin-orbit coupling. The presence of a strong magnetic field was suggested indeed by the typical magnetic activity 
signatures found in the photometric data, as the solution of the OGLE light curve (Paper I) required a large dark spot on the stellar surface of the primary component. We expected, therefore, to detect other typical activity indicators in the spectra.

In synchronized binaries the net effects of AML are shrinkage of the orbit, orbital period decrease and faster spinning of the components. The secular evolution of the orbital period is strictly related to the efficiency of magnetic braking.

At the time when the available data were rather sparse and inhomogeneous, the lack of close binaries with late (M) type components could be explained in terms of a selection effect against the discovery of intrinsically faint objects. However, the modern large surveys for variability (such as OGLE) have shown that the effect is real, as these systems are indeed very rare, even in large, homogeneous and at some level complete datasets.

The dearth of such systems implies then some evolutionary effect. Either, for some configuration, the orbital evolution should become so fast to prevent system detection or, on the contrary, it should be very slow. The latter hypothesis seems more likely in light of the current understanding of the AML processes in late-type single stars.

Studies of cluster (Terndrup et al. 2000, and references therein) and of field M dwarfs (Delfosse et al. 1998) of different ages favor the hypothesis of a spin-down time-scale that increases with decreasing mass (and show a saturation of the activity indicators with rotation). This evidence has been related to the properties of the $\alpha \Omega$ dynamo that operates at the interface between the radiative core and the convective envelope, and that is expected to work up to spectral type M3 (or may be later for magnetic dwarfs, see Mullan \& MacDonald 2001). The dynamo and the braking efficiency increase - at fixed radius - with faster rotation, until a "saturation" of the mean field is reached at rotational velocities that slightly depend on the sample, but are of the order of $10 \mathrm{~km} \mathrm{~s}^{-1}$. Looking instead at the behavior with spectral type, as the angular momentum is essentially extracted from the convective envelope, which deepens with advancing spectral type, later stars have longer spin-down timescales. Finally, in fully convective stars a further reduction of AML is expected, as the dynamo changes in nature (perhaps the small-scale turbulent dynamo of Durney et al. 1993).

In synchronized binaries with late type components, that at variance with single stars - rotate faster with increasing age, both effects contribute to lengthen the "contact" timescale. The studies of the period distribution of various samples of close binaries with solar-type components confirmed the abovementioned scenario, suggesting as well a saturated braking law (Rucinski 1983; Maceroni \& Van’t Veer 1991; Stępień 1995; Maceroni \& Rucinski 1999).

The expected physical properties of BW3 V38, as guessed in Paper I solely on the basis of the photometry, put the system exactly at the proposed border of the efficient braking and make the system of paramount importance for the study of magnetic braking mechanisms and the effects of fast rotation in late-type dwarfs. Its location in the C-M diagram is also of great interest, as our system lies exactly midway between the two - and for a long time the only - other known eclipsing binaries with late dwarf components, YY Gem and CM Dra, fundamental lower-MS calibrators, and next to its recently discovered, and presumably younger "twin”, CU Cnc (Delfosse et al. 1999).

Therefore, a spectroscopic study of this unique system was absolutely needed. In this paper we report the results of the observing runs aimed to get mid-resolution spectra of the binary. Section 2 describes the details of data acquisition and reduction, Sect. 3 presents the simultaneous solution of the light and radial velocity curves and a discussion of the system physical properties, Sect. 4 gives an outline of the possible orbital evolution, followed by the conclusions.

\section{The spectroscopic observations}

BW3 V38 is a rather difficult target for radial velocity determination. Its period requires short exposures to avoid Doppler smearing of spectral lines. Assuming, as a rule of thumb, that an acceptable exposure should last no more than $5 \%$ of the orbital period (i.e. 0.05 in phase), the maximum duration shall not exceed $900^{\mathrm{s}}$. Therefore, because of its faintness, a mediumlarge class telescope is required. Furthermore, the field is relatively crowded and the binary has a nearby (at $\sim 2^{\prime \prime}$ distance) and brighter (by $\sim 2 \mathrm{mag}$ ) optical companion.

BW3 V38 was observed at ESO - La Silla during two half nights, in August 2001 (a previously scheduled run in July 2000 was completely lost because of bad weather). A total of 17 long slit spectra were obtained, at various orbital phases, with the ESO $3.5 \mathrm{~m}$ New Technology Telescope (NTT) and the ESO Multi-Mode Instrument (EMMI) in the red midresolution spectrographic mode. The slit width was fixed at $1^{\prime \prime}$. The spectra are $640 \AA$ wide and are centered around the $\mathrm{H} \alpha$ line ( $\lambda=6563 \AA$ ). The selected grating (\#6) has a nominal resolution of 5500 (at $6000 \AA$ and with the $1^{\prime \prime}$-wide slit) and a dispersion of $12.5 \AA \mathrm{mm}^{-1}$ (or $0.3 \AA \mathrm{pix}^{-1}$ ). The spectra of a few other $\mathrm{K}-\mathrm{M}$ dwarfs, to be used as templates, were acquired as well; the stars were chosen from the lists of Kirkpatrick et al. (1991) and Henry et al. (1994). Finally, we selected the two templates that looked more similar to the spectra of the binary and of the optical companion, i.e. GJ 729 (M3.5 V) and GJ 775 (K5 V), for which accurate radial velocities were recently obtained by Nidever et al. (2002).

Because of the above-mentioned constraints the exposures were limited to $900^{\mathrm{s}}$ and the slit was aligned along the direction connecting our main target with the optical companion (and not - as usual - according to the parallactic angle). In this way the spectra of both objects could simultaneously be acquired and the contribution of the close-by star could be taken into account in data reduction, providing - as an extra bonus - a useful internal check.

The data reduction was performed by means of standard techniques (the long slit reduction routines of the IRAF ${ }^{1}$ package). The steps were bias subtraction, flat fielding, (partial) cosmic ray removal, one-dimensional spectrum extraction and

\footnotetext{
${ }^{1}$ IRAF is distributed by the National Optical Astronomical Observatories, which are operated by the Association of the Universities for Research in Astronomy, Inc., under cooperative agreement with the National Science Foundation.
} 


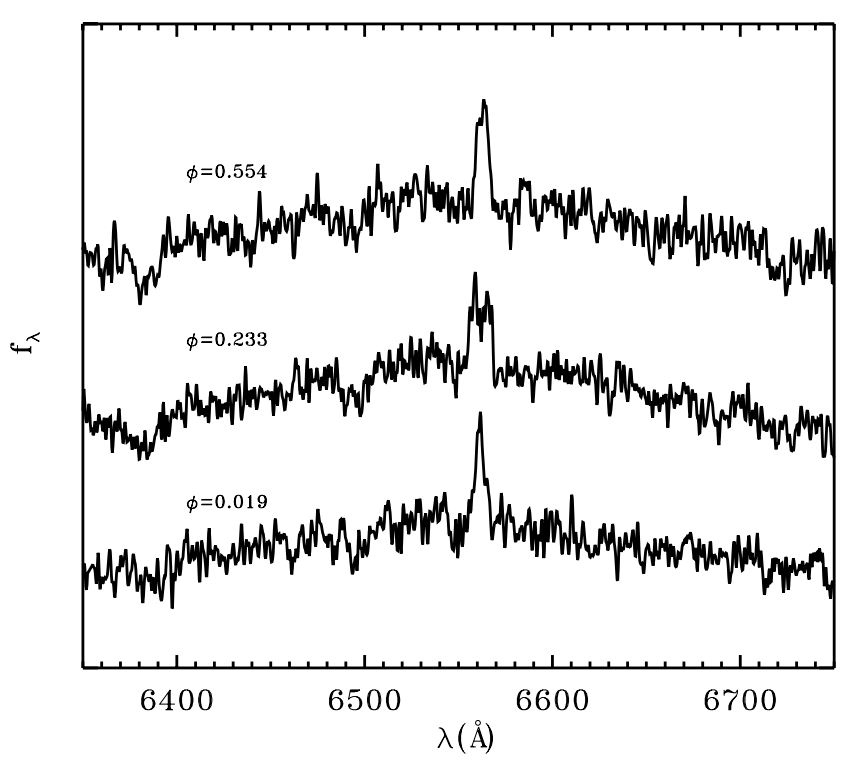

Fig. 1. Three smoothed spectra of BW3 V38, from bottom to top: around primary eclipse, quadrature and secondary eclipse. The spectra are normalized to the highest continuum level. The splitting of $\mathrm{H} \alpha$ at quadrature indicates the presence of strong chromosphere in both components.

wavelength calibration. The radial velocities were obtained by standard cross correlation technique (as provided by the IDL 5.5 and IRAF packages) after a few preliminary steps of: noise reduction (smoothing by convolution with a 3-pix FWHM Gaussian curve, i.e. smaller than the slit projection), normalization to a pseudo-continuum (obtained by means of the IRAF continuum polynomial fitting and rejection algorithms) and transformation to the heliocentric system. The orbital phase determination showed an excellent agreement with the ephemeris published by Udalski et al. (1995); in fact the same binary was briefly observed during the second phase of the OGLE experiment, a few years later, and no measurable period change or phase shift was found (Udalski, private communication).

At this stage, an inspection of the radial velocities of the optical companion revealed the existence of a non-negligible nightly positive trend in the derived velocities (with a range of $\simeq 30 \mathrm{~km} \mathrm{~s}^{-1}$ ), corresponding to $\sim 2$ pixels. While its origin remained unknown, but could presumably be ascribed to mechanical problems, the trend could be removed completely by a careful use of the night sky emission lines. The dispersion solution was recomputed, using for wavelength reference the night-sky lines, which could be identified using the spectral atlas of $\mathrm{OH}$ and $\mathrm{O}_{2}$ emission lines by Osterbrock et al. (1996). Each spectrum was wavelength-calibrated with its own sky lines (with the exclusion of the bright template stars, whose exposure were too short to get a usable sky level; in that case the nearest exposure of the binary was used). In this way the trend completely disappeared and the standard deviation of the radial velocity of the optical companion decreased from 9.3 to $2.2 \mathrm{~km} \mathrm{~s}^{-1}$.

Figure 1 shows three spectra of the variable close to the two minima and to quadrature. The typical $\mathrm{S} / \mathrm{N}$ ratio of the binary spectra is $\sim 10$, i.e. still acceptable for radial velocity
Table 1. Simultaneous light and radial velocity curve solution of BW3 V38.

\begin{tabular}{lll}
\hline \hline Parameter & This paper & Paper I \\
\hline$i($ degr $)$ & $85.51 \pm 0.84$ & $85.7 \pm 1.2$ \\
$q$ & $0.950 \pm 0.067$ & $0.77 \pm 0.09$ \\
$\Omega_{1}$ & $3.71 \pm 0.11$ & $3.48 \pm 0.13$ \\
$\Omega_{2}$ & $4.02 \pm 0.19$ & $3.55 \pm 0.25$ \\
$T_{2}$ & $3448 \pm 11$ & $3459 \pm 15$ \\
$\left(T_{1}=3500\right)$ & & \\
$L_{1} /\left(L_{1}+L_{2}\right)$ & $0.594 \pm 0.031$ & $0.597 \pm 0.036$ \\
$\Delta \phi$ & $0.0023 \pm 0.0005$ & $0.0020 \pm 0.0006$ \\
\hline$r_{1}($ side $)$ & $0.372 \pm 0.008$ & $0.380 \pm 0.011$ \\
$r_{2}(\mathrm{side})$ & $0.323 \pm 0.028$ & $0.322 \pm 0.050$ \\
\hline$A\left(R_{\odot}\right)$ & $1.355 \pm 0.066$ & \\
$K_{1}\left(\mathrm{~km} \mathrm{~s}^{-1}\right)$ & $167 \pm 8$ & \\
$\gamma\left(\mathrm{km} \mathrm{s}^{-1}\right)$ & $-0.6 \pm 5.9$ & \\
\hline
\end{tabular}

determination. However, the task was not expected to be easy, because of the late spectral type and the related problems of continuum normalization, and because of the high rotational velocity of the components.

On the basis of Fig. 1 and of the parameters we know from the light curve solution of Paper I (in particular the high inclination that assures almost total eclipses), we can conclude that the binary is indeed formed by two active similar late-type dwarfs. While we do not have in our spectral range features that allow a quantitative spectral classification (as, for the red part of a late dwarf spectrum, the TiO5 index of Reid et al. 1995), the general shape of the spectrum and the visibility of the TiO bands at 6569 and $6651 \AA$ suggest a M3 V classification.

The second firm conclusion we can draw from the figure is that both components are dMe stars, as a splitting of $\mathrm{H} \alpha$ in emission is clearly visible at quadrature. For both components the $\mathrm{H} \alpha$ equivalent width around quadrature is $\simeq 2.7 \AA$, a value indicating the presence of strong chromospheres. Among dwarfs with $\mathrm{H} \alpha$ in emission, however, higher $E W$ values are found, especially in components of wider (and presumably younger) binary systems (Gizis et al. 2002), typical values span the range $0-10 \AA$.

The age of BW3 V38 is unknown, as well as its population characteristics; however its location in the galactic plane (Paper I) and the low value of at least the barycentric velocity (see Table 1) offer a weak support for a young/intermediate disk population. Regardless, our system must be significantly older than the very similar but wider system CU Cnc (GJ 2069A). This too is formed by two M3 dwarfs, but has an orbital period of 2.77 and an estimated age, from the likely association with the Castor moving group, of only $\simeq 300 \mathrm{My}$ (Ribas 2003, hereafter R03). According to Gizis et al. (2002), the $\mathrm{H} \alpha E W$ of $\mathrm{CU} \mathrm{Cnc}$ is $\cong 4.6 \AA$ and its activity level, as estimated from the ratio $L_{\mathrm{H} \alpha} / L_{\mathrm{bol}}$, is very high. R03 reports individual $E W$ values for each component (of 3.85 and $4.05 \AA$ for the primary and the secondary) and a value of $L_{x} / L_{\text {bol }} \cong 10^{-3}$, that presumably marks the activity saturation limit (Delfosse et al. 1998). 


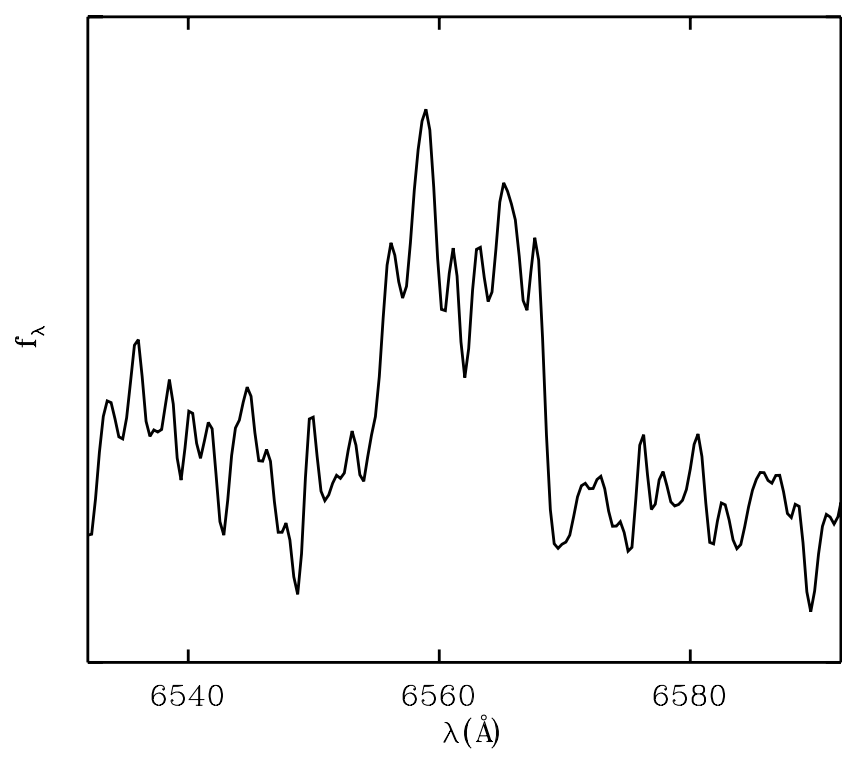

Fig. 2. Detail of the $\mathrm{H} \alpha$ region in the spectrum at phase $\phi=0.233$, note the partial reversal of the profiles.

The shape of the $\mathrm{H} \alpha$ profile of BW3 V38, that around quadrature clearly shows a partial central reversal for both components (Fig. 2), indicates a lower activity level than typically observed in most close binaries containing dMe dwarfs, which usually show a peaked profile (Stauffer \& Hartmann 1986).

Figure 3 shows the radial velocity curves, as derived by the cross correlation technique. The cross correlation functions were fitted with Gaussian curves, leaving as free parameters the position and height. The amplitudes were estimated from the fit close to minimum phases (the light curve solution indicates almost total eclipses of two similar size components) and kept constant for the other orbital phases. As the least square fitting routine provided, as usual, unrealistically small errors, a value between 20 and $30 \mathrm{~km} \mathrm{~s}^{-1}$ (depending on the component and phase) was estimated by means of the IRAF implementation of the Tonry \& Davis (1979) algorithm, where the velocity errors are also computed, based on the fitted peak height and the antisymmetric noise. This estimate tells us that the accuracy of the derived orbital elements will not be high enough to provide a - badly needed - improvement of the lower main sequence calibration. However, a full exploitation of the existing data will improve our knowledge of this unique binary system.

\section{Physical properties of BW3 V38}

A simultaneous solution of the radial velocity curves and the OGLE I-band light curve was computed by means of the Wilson and Devinney code (Wilson \& Devinney 1971, in its 2001 distribution $^{2}$ ). The input parameters for the light curve solution were taken from Paper I and those for the radial velocity curves were obtained by a preliminary sine fit of the velocity curves. Looking for further information on the component color and temperature, we checked if the system was detected in the 2MASS survey (Skrutskie et al. 1995), but the

\footnotetext{
2 The code is kindly made available by the author at http://www. astro.ufl.edu/pub/wilson
}

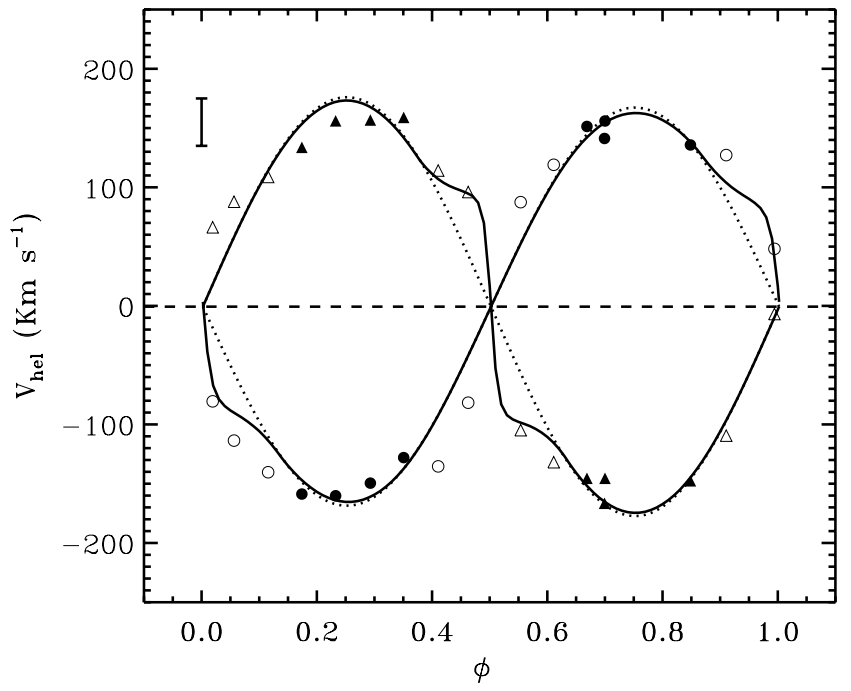

Fig. 3. The radial velocity curve of BW3 V38. Dots indicate the primary and triangles the secondary velocity curve, the full line the result of the simultaneous solution, the dotted line the stellar barycentric radial velocities. Empty symbols correspond to points that were given half weight in the simultaneous solution. The vertical bar is the mean uncertainty of the observed points.

only source at the appropriate location corresponds to a K-type star, i.e. to the brighter optical companion (unfortunately its optical separation with the binary is comparable to the survey true PSF resolution of $\simeq 2.5^{\prime \prime}(F W H M)$ ). We kept therefore the primary effective temperature derived in Paper I.

As in Paper I, the photometric observations were given equal weights, while the radial velocities were unequally weighted $\left(w_{i}=0.5\right.$ for phases close to the photometric minima, see Fig. 3). The curve dependent weights (required to weigh the different sets of observations with respect to each other) were assigned, respectively, on the basis of the standard deviation of the preliminary sine fit for the radial velocity curves and of the local fit at maxima for the light curve. The corresponding values are $\sigma_{\mathrm{rv}}=25 \mathrm{~km} \mathrm{~s}^{-1}$ and $\sigma_{\mathrm{lc}}=0.02$ (in light units). The iterative solution used the multiple subset scheme (Wilson \& Biermann 1976). The adjusted parameters were the orbital inclination, $i$, the mass ratio $^{3}, q=m_{2} / m_{1}$, the surface reduced potentials, $\Omega_{1,2}$, the secondary effective temperature, $T_{2}$, the primary fractional luminosity, $L_{1} /\left(L_{1}+L_{2}\right)$, the orbit semiaxis, $A$, the barycentric velocity $\gamma$, and the phase shift $\Delta \phi$. The final solution is shown Figs. 3, 4 and the final parameter values are collected in Table 1. The latter contains as well the component "side" fractional radii (i.e. the fractional radii in the orbital plane and at $90^{\circ}$ from the axis connecting the star barycenters) and the primary radial velocity curve amplitude, $K_{1}$. The other non-adjusted parameters defining the solution maintained the same values of Paper I, namely the linear limb darkening coefficients $x_{1,2}=0.56$ at $\lambda=7520 \AA$ (and $x_{1,2}=0.65$ at $\mathrm{H} \alpha$ ), the gravity darkening coefficients $\beta_{1,2}=0.32$, the albe$\operatorname{dos} a_{1,2}=0.5$. Moreover, the dark spot of Paper I was kept

\footnotetext{
3 Star " 1 " is defined in the code as the component eclipsed at primary minimum, in our case $q<1$ and star " 1 ", the primary, is as well the more massive component.
} 

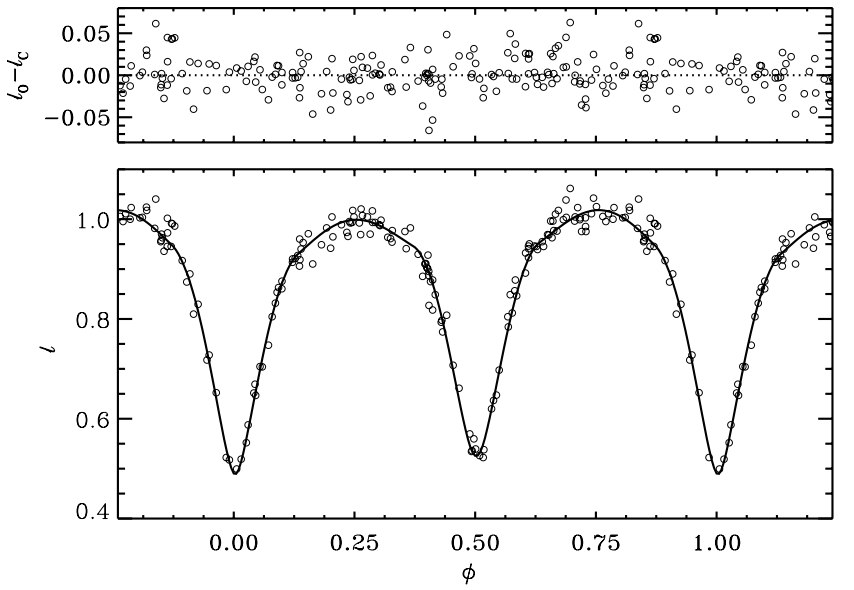

Fig. 4. The new light curve solution BW3 V38 (lower panel) and the deviations. Paper I solution is virtually indistinguishable from the new one and was not drawn, to avoid confusion.

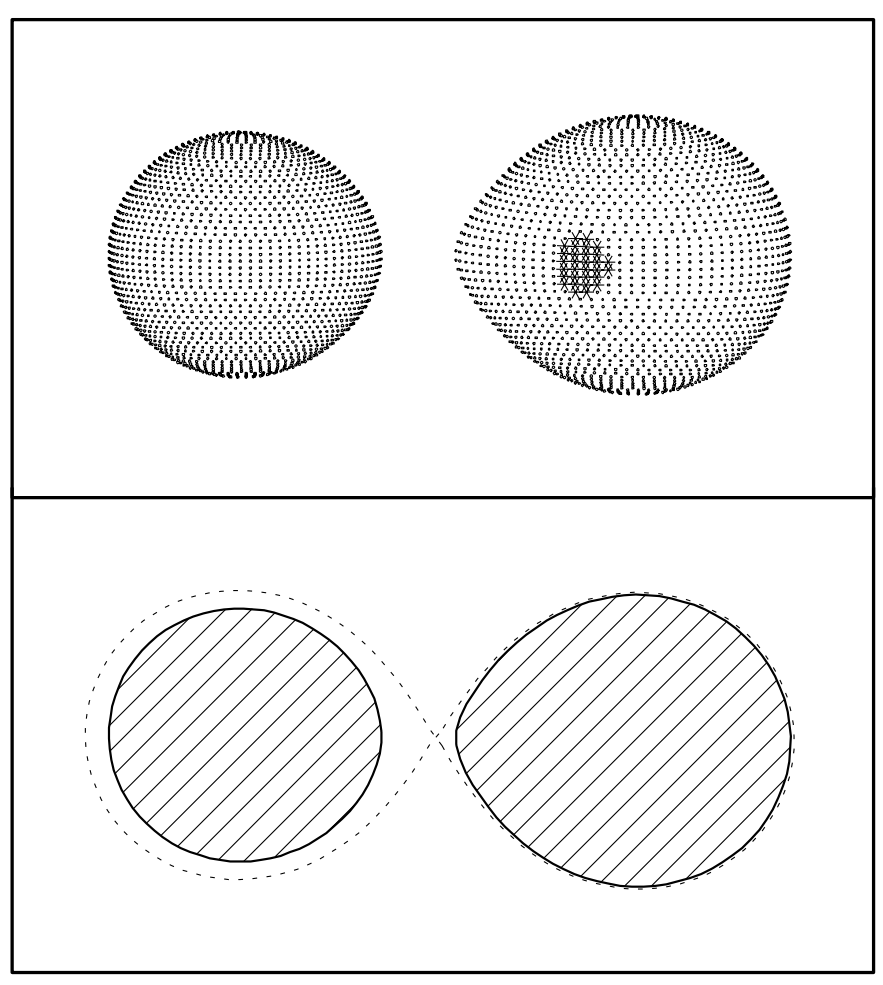

Fig. 5. The 3-D drawing at orbital phase 0.25 (upper panel) and the equatorial section (lower panel) of BW3 V38. The dashed line in the lower panel indicates the critical inner Roche lobe.

unchanged in the simultaneous solution and is defined by longitude and angular radius, respectively of 290 and 11 degrees, and the temperature factor with respect to the unspotted surface, $T_{\mathrm{s}} / T_{*}=0.8$. All the errors reported in the tables are standard errors.

The introduction of the radial velocity curves in the solution slightly changes the system configuration (Fig. 5) with respect to that obtained in Paper I. The most affected parameter is - as one could expect - the mass ratio, that increases from $q=0.77$ to $q=0.95$. The other adjusted parameters vary as predicted by the correlation plots of Paper I, that were computed by means
Table 2. Derived physical quantities for BW3 V38 components.

\begin{tabular}{lcc}
\hline \hline $\begin{array}{l}\text { Parameter } \\
\text { (solar units) }\end{array}$ & Primary & Secondary \\
\hline$m$ & $0.44 \pm 0.07$ & $0.41 \pm 0.09$ \\
$R$ & $0.51 \pm 0.04$ & $0.44 \pm 0.06$ \\
$M_{\text {bol }}^{a}$ & $8.39 \pm 0.08$ & $8.78 \pm 0.13$ \\
$\log g$ & $4.66 \pm 0.13$ & $4.77 \pm 0.21$ \\
\hline
\end{tabular}

${ }^{a}$ Based on $M_{\mathrm{bol}, \odot}=4.75$.

of bootstrap "resampling". The configuration is still a detached one, but the primary is slightly closer to the critical lobe (the ratio between the mean stellar radius and that of the critical surface increases from $r / r_{R}=0.95$ to $\left.r / r_{R}=0.98\right)$, while the secondary is somewhat more inside $\left(r / r_{R}\right.$ decreases from 0.90 to 0.86$)$. The increase in relative radius is balanced by the decrease of secondary temperature, so that the fractional luminosities are almost unchanged.

It is well known that in detached and partially eclipsing systems the light curve shape is only weakly dependent on the mass ratio and that there is a rather strong correlation between the geometric parameters. This explains why the maximum deviation between the new and the Paper I synthetic light curves, corresponding to the two solutions, is of only $0.005 \mathrm{mag}$ (and the mean difference of just $0.001 \mathrm{mag}$ ).

The derived absolute dimensions are presented in Table 2. The uncertainties on the physical parameters are unfortunately large, nevertheless the location of the components in the H-R and in the mass radius diagrams (Fig. 6) are consistent with those of typical M-dwarfs.

Figure 6 shows a comparison between the location of the known double-lined eclipsing binaries with $\mathrm{M}$ dwarf components and three theoretical isochrones of different authors (D'Antona 2004; Baraffe et al. 1998; Yi et al. 2001).

The binary systems are: the already mentioned CU Cnc (R03), CM Dra (Lacy 1977; Metcalfe et al. 1996) YY Gem and V818 Tau (Torres \& Ribas 2002), whose secondary component is an $\mathrm{M}$ dwarf. It has to be stressed that CM Dra is a non-homogeneous system with respect to the rest of the sample (and to the theoretical isochrones), as it seems to be a population II object (Lacy 1977). The physical parameters of the various objects are taken from the mentioned papers.

The isochrones are for an age of $1 \mathrm{~Gy}$ and solar chemical composition; our system is probably older, but evolution affects only slightly the isochrone location for the mass range of interest (the isochrone age was actually chosen as a compromise between the age of the other younger systems and our object). All the other systems but CM Dra are believed indeed to be younger: $\mathrm{CU} \mathrm{Cnc}$ because of the association, on the basis of its space motion, with the Castor moving group, to which YY Gem presumably belongs, and V818 Tau as being a member of the Hyades cluster.

Though the physical parameters of BW3 V38 are not accurate enough to pose constraints on the lower main sequence calibration, its location on the mass-radius diagram is - generally - consistent with the conclusions drawn from all the other 

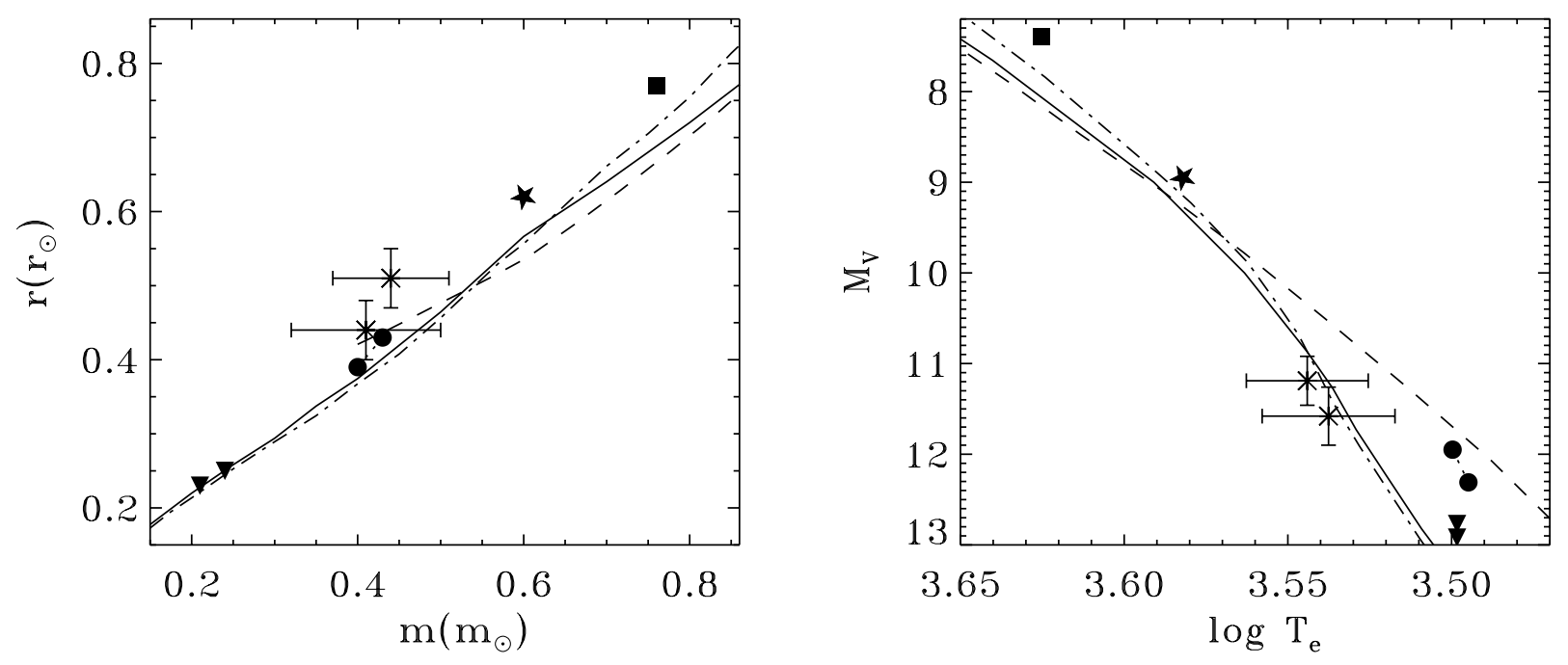

Fig. 6. The location of BW3 V38 components (points with error bars) in the mass radius and in the $\log T_{\mathrm{e}}-M_{V}$ diagrams. The locations of the other known eclipsing binaries with M-dwarf component(s) are also shown: the filled dots indicate CU Cnc components (R03), the starred dot the secondary star of V818 Tau, the square YY Gem mean conponent (TR02), the triangles CM Dra components (Lacy 1977; Metcalfe et al. 1996). The theoretical isochrones (1 Gy, solar chemical composition) are from D'Antona (2004) (continuous line), Baraffe et al. (1998) (dashed-dotted line) and Yi et al. (2001) (dashed line).

systems, i.e. that the theoretical models seem to underestimate the MS stellar radii and/or overestimate the effective temperatures. A comparison with a larger set of models, performed by Ribas (R03) and Torres \& Ribas (2002) shows that this underestimate can be as high as $20 \%$ for the stellar radius, and that most models overestimate temperatures (by $150 \mathrm{~K}$ ). This is regardless of the recent improvements in the input physics for low mass stars. (The apparent agreement, in Fig. 6, of CM Dra parameters is obviously due to the difference in chemical composition of the object with respect to that of the theoretical isochrones; lower metallicity isochrones would correspond to smaller radii.)

The radiative properties of BW3 V38 seem in good agreement with those of the other dwarfs in the same mass range, however, at variance with CU Cnc, the temperatures could not be independently derived.

Both BW3 V38 components are very fast rotators: the radii of Table 2 and the hypothesis of spin-orbit synchronization yield equatorial velocities of, respectively, $v_{1}=131 \pm 10$ and $v_{2}=113 \pm 15 \mathrm{~km} \mathrm{~s}^{-1}$.

Mullan \& MacDonald (2001) propose that the larger radii and lower temperatures of observed $\mathrm{M}$ dwarfs are due to the presence of strong magnetic fields. There is the indication, indeed, that active dMe dwarfs $(V-I<2.7)$ are brighter on average by about 0.5 mag than $\mathrm{dM}$ stars of the same color (Hawley et al. 1996), and that the excess is even larger in clusters: $0.7 \mathrm{mag}$ in the Hyades and $1.2 \mathrm{mag}$ in IC 2602. The nonstandard stellar models of Mullan \& MacDonald (2001), that include a simplified treatment of magnetic field effects, agree with these observations, suggesting larger radii and lower temperatures for stars hosting strong fields (up to several tens of MGauss at the base of the convective zone). In addition in their models the limit of transition to a fully convective structure is shifted to masses lower than the standard model typical value $0.35 / 0.40 M_{\odot}$. As a consequence the $\alpha \Omega$ dynamo can still be at work for these mass values, and the AML through magnetic braking as well. The reason of the dearth of close systems with $\mathrm{M}$ type components shall therefore be found in the timescale of the AML process, rather than in its inhibition.

\section{An outline of BW3 V38 orbital evolution}

With the physical parameters of Table 2 the angular momentum content of BW3 V38 is $H_{\text {orb }}=1.3 \times 10^{51}$ in cgs units (without the spin AM that amounts anyway to $\leq 10^{50} \mathrm{cgs}$, i.e. less than $10 \%$ ). This is presumably only a fraction of the original content.

There are good reasons to believe that systems like BW3 V38 form with a much larger separation and evolve, later on, toward closer orbits. The current knowledge of close binary formation (Ghez 1996; Bonnell 2001) rules out the fission process that does not seem able to produce stable binary configurations. The other "in situ" formation process, capture and hardening of the binary by ejection of a component from a triple system, is rare and inefficient in the field and works against the presence of small mass stars in the close pair, as it is generally the less massive star that is ejected.

The hydrodynamical calculations of binary formation by fragmentation of Bate et al. (2002) indicate that proto-binaries form with large separations ( $\geq 10 \mathrm{AU}$ ) and go through a phase of accretion and orbital evolution towards tighter orbits ( $\sim 1$ AU). Subsequent less known phases of orbital evolution, presumably due to interaction with the matter still present in the system, should finally produce really close binaries.

On observational grounds, Melo et al. (2001) report in their collection of data for known PMS spectroscopic binaries that the shortest orbital period (relevant to a G5+K1 system) is $P=1.67$.

As a thorough description of the formation processes from the initial cloud to the Pre Main Sequence (PMS) is not yet 
available, a rough estimate of a minimum separation can be derived by the simple argument of the orbital semi-axis necessary to accommodate the pre-main-sequence (PMS) progenitors of the binary. The PMS tracks of D'Antona \& Mazzitelli (1994) for a $0.4 M_{\odot}$ star give a radius, during the D-burning phase, of $\sim 3 R_{\odot}$, corresponding to a period of $\sim 1$ d. 5 .

Several authors have computed the orbital period evolution of a synchronized binary under the influence of AML (Vilhu 1982; Rucinski 1983; Maceroni \& Van't Veer 1991; Maceroni 1992; Stępień 1995; Maceroni \& Rucinski 1999) developing an initial suggestion by Van't Veer (1979). Most works focused on deriving the slope of the braking law, without directly computing the amount of AML. Moreover, the greatest part of the abovementioned works concerns the better known case of systems with solar-type components. We can try at least to estimate the expected evolution of the orbital period using the available data on $\mathrm{M}$ dwarfs.

The spin-down of a single late-type star can be described by:

$\dot{\omega}=-c_{\text {sd }} \omega^{\alpha}$

where $c_{\mathrm{sd}}$ and $\alpha$ are constants and $\omega$ is the angular velocity. An elementary integration yields $(\alpha \neq 1)$ :

$\omega=\omega_{\mathrm{o}}\left(1-\frac{t-t_{\mathrm{o}}}{(1-\alpha) \tau_{\mathrm{sd}}}\right)^{1 /(1-\alpha)}$

where the quantity

$\tau_{\mathrm{sd}}=\left[\frac{\omega}{\dot{\omega}}\right]_{\mathrm{t}=\mathrm{t}_{\mathrm{o}}}=\frac{\omega_{\mathrm{o}}^{1-\alpha}}{c_{\mathrm{sd}}}$

is the spin-down timescale (for $t=t_{\mathrm{o}}$ ). In a close binary thanks to synchronization (which can be considered as instantaneous, taking place on a much shorter timescale than AML, see for instance Maceroni \& Van't Veer 1991) the loss of spin angular momentum from both components:

$\dot{H}_{\text {sp }}=k^{2}\left(m_{1} r_{1}^{2}+m_{2} r_{2}^{2}\right) \dot{\omega}=-k^{2}\left(m_{1} r_{1}^{2}+m_{2} r_{2}^{2}\right) c_{\text {sd }} \omega^{\alpha}$

can be equated to the orbital angular momentum loss:

$\dot{H}_{\text {orb }}=-\frac{1}{3} G^{2 / 3} q(1+q)^{-2} m_{\mathrm{T}}^{5 / 3} \omega^{-4 / 3} \dot{\omega}$

with $m_{\mathrm{T}}$ being the total mass. It is assumed that $\omega_{\text {orb }} \equiv \omega_{\text {sp }} \equiv \omega$, and that the masses, the radii and the gyration radius, $k^{2}$, do not appreciably change with time. Besides, the small contribution of the spin to the total AM (the quantity to which Eq. (5) should apply) is neglected.

From Eqs. (4), (5), and introducing the orbital period, we get:

$\dot{P}=-c P^{2 / 3-\alpha}$

with

$c=\frac{3(2 \pi)^{\alpha+1 / 3} k^{2}\left(m_{1} r_{1}^{2}+m_{2} r_{2}^{2}\right) c_{\mathrm{sd}}(1+q)^{2}}{G^{2 / 3} m_{\mathrm{T}}^{5 / 3} q}$.

Equation (6) can be integrated once suitable values for $\alpha, c_{\mathrm{sd}}$ and $k^{2}$ are chosen.
Delfosse et al. (1998) derive that the spindown timescale of field M dwarfs at spectral type M4 is "a significant fraction of the age of the young disk ( $3 \mathrm{~Gy})$ ", a reasonable choice for M3 components is $\tau_{\text {sd }} \simeq 1 \mathrm{~Gy}$.

To derive $c_{\text {sd }}$ from Eq. (3) we also need a value for $\omega_{0}$. The abovementioned paper gives the observed shortest rotation pe$\operatorname{riod}(P / \sin i)$ of a field M4 dwarf belonging to the young disk population (G 165-08), its value being about $7^{\mathrm{h}}$. This value is derived from $v \sin i$ measurements, but it is reasonable to assume that the fastest observed rotation corresponds as well to high inclination. In the following we also computed, as a check, an extreme case with a very low inclination value.

To choose $\alpha$ we recall that both the studies on single stars and on binaries with late type components indicate saturation of the braking law. The saturation boundary at $v \geq 10 \mathrm{~km} \mathrm{~s}^{-1}$ corresponds, with the radii of BW3 V38 components, to an orbital period $\sim 2$ d 6 , meaning that most if not all of its period evolution must have taken place in a saturated regime. Maceroni \& Rucinski (1999), from a study of the period distribution of late type binaries with equal components selected from the OGLE I database, find a "best value" at $\alpha=0.25$. Previous studies, (as Maceroni \& Van't Veer 1991; Maceroni 1992), suggest $\alpha \simeq 0$. The models and the analysis of $\mathrm{M}$ dwarf data suggest similar slopes, with, this in case, a shift of the saturation boundary to longer periods with decreasing mass (Sills et al. 2000; Pizzolato et al. 2003). Finally, the value of $k^{2}$ does not vary much with mass along the main sequence, going from $k^{2}=0.1$ for a solar type star to $k^{2}=0.2$ for a fully convective brown dwarf (Armitage \& Bonnell 2002).

Equation (6) was integrated with the different sets of parameters of Table 3 and the results are shown in Fig. 7. The curves were obtained integrating the equation backwards in time and taking as a boundary condition the present period of BW3 V38. The figure gives, therefore, as function of the system age, the initial period from which our system could have evolved to the present configuration. The extreme case with $i=30^{\circ}$ corresponds of course to the fastest evolution. The value of $\tau_{\text {sd }}$ was kept fixed, but any larger value would make the evolution still slower.

In all cases period decrease is rather slow. The evolution of a system similar to $\mathrm{CU}$ Cnc (i.e., $P_{\text {in }}=2$ d.77) would take, to reach the BW3 V38 configuration, a time larger than the age of the Universe (assumed to be $14 \mathrm{~Gy}$ ).

One may wonder if other AML mechanisms, such as AML by gravitational radiation, could have significantly contributed to the orbital evolution of the system. The time-scale of period change due to gravitational waves can be estimated from (Press $\&$ Thorne 1972):

$\tau_{\mathrm{G}}=\left|\frac{P}{\dot{P}}\right|=2.8 \times 10^{7} \frac{\left(m_{1}+m_{2}\right)^{1 / 3}}{m_{1} m_{2}} P^{8 / 3}$

with $\tau_{\mathrm{G}}$ in years and $P$ in hours. For an initial period of the order of a day (and using the masses from Table 2), we get $\tau_{\mathrm{G}} \simeq 7 \times 10^{11} \mathrm{y}$, while, for the current period value, $\tau_{\mathrm{G}} \simeq 9.4 \mathrm{~Gy}$.

For comparison purposes, a timescale of AML via magnetic braking, $\tau_{\mathrm{M}}=|P / \dot{P}|$, can readily be derived from Eq. (6) and its value, for the current period, varies in the range $2.7-9.8 \mathrm{~Gy}$ 


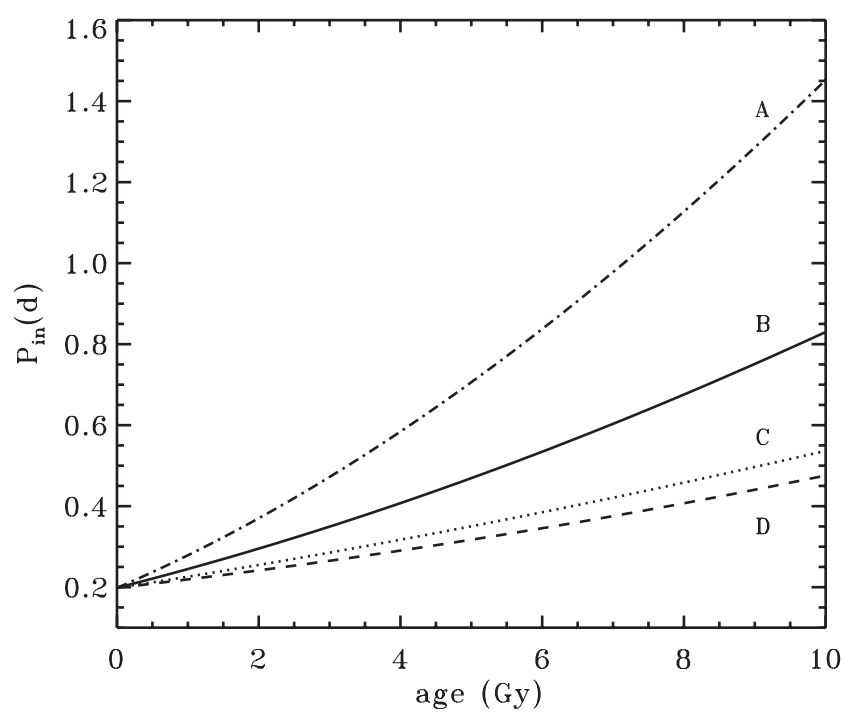

Fig. 7. The initial period, as function of system age, that is required to reach by AML the present period of BW3 V38, according to the scheme proposed in the text. The curves correspond to different values of the parameters entering the computation, as indicated in Table 3.

Table 3. Orbital evolution parameters.

\begin{tabular}{lllll}
\hline \hline Model & $\alpha$ & $k^{2}$ & $i$ & $\tau_{\mathrm{M}}(\mathrm{Gy})$ \\
\hline $\mathrm{A}$ & 0.25 & 0.2 & $30^{\circ}$ & 2.7 \\
$\mathrm{~B}$ & 0.25 & 0.2 & $90^{\circ}$ & 4.5 \\
$\mathrm{C}$ & 0.25 & 0.1 & $90^{\circ}$ & 8.9 \\
$\mathrm{D}$ & 0.00 & 0.1 & $90^{\circ}$ & 9.8 \\
\hline
\end{tabular}

(last column of Table 3). The contribution of gravitational radiation could, therefore, be relevant in the future orbital evolution of the system, and only in the cases where the magnetic braking has the minimum efficiency. However - given the steep dependence of $\tau_{\mathrm{G}}$ on $P$ - its contribution was negligible during the past evolution of the binary.

In conclusion, in the outlined scenario, if BW3 V38 has an age typical of an intermediate disk population (say, 5 Gy) its initial period could not have exceeded $P_{\text {in }} \sim 1^{\mathrm{d}}$ and, even with all the uncertainties related to the AML description, it is not surprising that systems similar to it are very rare.

\section{Conclusions}

The analysis of the spectroscopic data of BW3 V38, in spite of their moderate quality, significantly increases our knowledge of this interesting system.

The classification of the components as M3 dwarfs (which was previously based only on the $V-I$ color from OGLE-I) is now confirmed. Both components are indeed dMe stars, with strong chromospheres, as indicated by the $\mathrm{H} \alpha$ emission profiles. The $\mathrm{H} \alpha$ equivalent widths suggest an average activity level, when compared to similar binaries. This is an indication that the system is older than similar systems. Thanks to BW3 V38 we have clear evidence that binaries with M dwarfs and very short periods are rare objects but do exist. If they form by AML from wider orbits, the AML timescale will then be $\leq$ the age of the Galaxy.

The simultaneous radial and light curve solution provided a consistent set of absolute elements. The components are very similar stars, with the slightly more massive one very close to the limiting Roche surface. The system can be considered a tighter twin of CU Cnc.

The derived masses and radii agree - within the limits of the large uncertainty - with the relations obtained from the M-type components of the other known systems, which indicate a discrepancy, of the order of $10 \%$, between the theoretical models and the values determined from eclipsing binary stars. It has been suggested that the effect could be related to the presence of strong magnetic fields, as dMe stars are indeed brighter than the inactive dM dwarfs. In addition the BW3 V38 components are very fast rotators $\left(130 \mathrm{~km} \mathrm{~s}^{-1}\right)$ and the larger radii could also be related to this property.

A simple estimate of the orbital evolution of the system, taking into account the latest findings on the spin down of late type dwarfs, shows that the AML loss process is rather slow (the evolution from a configuration similar to that of CU Cnc would take, in the fastest case, $\sim 14 \mathrm{~Gy}$ ). In this frame the rareness of binaries similar to BW3 V38 can easily be understood.

It is clear that the system deserves better quality observations. It could be a fundamental system to better constrain the lower MS calibration, and to study the effects of magnetic fields and fast rotation, but higher $\mathrm{S} / \mathrm{N}$ spectra are needed. These will require a telescope of the $8 \mathrm{~m}$ class. We hope that the results obtained with this study will trigger the interest in this unique object, and qualify it as a priority target for the immediate future.

Acknowledgements. The collection of data for this system was particularly difficult, as weather and technical problems impaired several efforts in this direction. C.M. especially thanks Slavek Rucinski, for continuous encouragement and for his help during a first attempt at CFHT, that unfortunately did not produce any useful photons. The team of ESO-NTT is also acknowledged for precious advice during and after the two observing runs at ESO. The authors are also indebted to Vincenzo Testa and Gian Luca Israel for discussions on data reduction techniques, to Francesca D'Antona for providing new isochrones and to the anonymous referee for useful comments. This research project was funded by MIUR/Cofin and F-INAF programs and by ESA-Prodex under contract 15448/01/NL/SFe(IC)-C90135.

\section{References}

Alvarez, R., \& Plez, B. 1998, A\&A, 330, 1109

Armitage, P. J., \& Bonnell, I. A. 2002, MNRAS, 330, L11

Baraffe, I., Chabrier, G., Allard, F., \& Hauschildt, P. H. 1998, A\&A, 337,403

Bate, M. R., Bonnell, I. A., \& Bromm, V. 2002, MNRAS, 336, 705

Bonnell, I. A. 2001, in The Formation of Binary Stars, ed. H. Zinnecker, \& R. D. Mathieu (Kluwer), IAU Symp., 200, 23

D’Antona, F., \& Mazzitelli, I. 1994, ApJS, 90, 467

D'Antona, F. 2004, private communication

Delfosse, X., Forveille, T., Perrier, C., \& Mayor, M. 1998, A\&A, 331, 581 
Delfosse, X., Forveille, T., Mayor, M., Burnet, M., \& Perrier, C. 1999, A\&A, 341, L63

Durney, B. R., De Young, D. S., \& Roxburgh, I. W. 1993, Sol. Phys., 145, 207

Ghez, A. M. 1996, in Evolutionary Processes in Binary Stars, NATO ASIC Proc., 477, 1

Gizis, J. E., Reid, I. N., \& Hawley, S. L. 2002, AJ, 123, 3356

Hawley, S. L., Gizis, J. E., \& Reid, I. N. 1996, AJ, 112, 2799

Henry, T. J., Kirkpatrick, J. D., \& Simons, D. A. 1994, AJ, 108, 1437

Kim, Y., \& Demarque, P. 1996, ApJ, 457, 340

Kirkpatrick, J. D., Henry, T. J., \& McCarthy, D. W. 1991, ApJS, 77, 417

Lacy, C. H. 1977, ApJ, 218, 444

Maceroni, C. 1992, in Inside the star, ed. W. W. Weiss, \& A. Baglin, ASP Conf., 40, 374

Maceroni, C., \& Rucinski, S. M. 1997, PASP, 109, 782

Maceroni, C., \& Rucinski, S. M. 1999, AJ, 118, 1819

Maceroni, C., \& Van't Veer, F. 1991, A\&A, 246, 91

Maceroni, C., \& Van't Veer, F. 1993, A\&A, 277, 515

Melo, C. H. F., Covino, E., Alcalá, J. M., \& Torres, G. 2001, A\&A, 378,898

Metcalfe, T. S., Mathieu, R. D., Latham, D. W., \& Torres, G. 1996, ApJ, 456, 356

Mochnacki, S. W. 1981, ApJ, 245, 650

Mullan, D. J., \& MacDonald, J. 2001, ApJ, 559, 353

Nidever, D. L., Marcy, G. W., Butler, R. P., Fischer, D. A., \& Vogt, S. S. 2002, ApJS, 141, 503
Noyes, R. W., Hartmann, L. W., Baliunas, S. L., Duncan, D. K., \& Vaughan, A. H. 1984, ApJ, 279, 763

Osterbrock, D. E., Fulbright, J. P., Martel, A. R., et al. 1996, PASP, 108, 277

Pizzolato, N., Maggio, A., Micela, G., Sciortino, S., \& Ventura, P. 2003, A\&A, 397, 147

Press, W. H., \& Thorne, K. S. 1972, ARA\&A, 10, 335

Reid, I. N., Hawley, S. L., \& Gizis, J. E. 1995, AJ, 110, 1838

Ribas, I. 2003, A\&A, 398, 239

Rucinski, S. M. 1983, The Observatory, 103, 280

Rucinski, S. M. 1992, AJ, 103, 960

Rucinski, S. M. 1997, AJ, 113, 407

Sills, A., Pinsonneault, M. H., \& Terndrup, D. M. 2000, ApJ, 534, 335

Skrutskie, M. F., Beichman, C., Capps, R., et al. 1995, BASS, 27, 1392

Stauffer, J. R., \& Hartmann, L. W. 1986, ApJS, 61, 531

Stępień, K. 1995, MNRAS, 274, 1019

Torres, G., \& Ribas, I. 2002, ApJ, 567, 1140

Terndrup, D. M., Stauffer, J. R., Pinsonneault, M. H., et al. 2000, AJ, 119,1303

Tonry, J., \& Davis, M. 1979, AJ, 84, 1511

Udalski, A., Szymański, M., Kałużny, J., et al. 1995, AcA, 45, 1

Van't Veer, F. 1979, A\&A, 80, 287

Vilhu, O. 1982, A\&A, 109, 17

Wilson, R. E., \& Devinney, E. J. 1971, ApJ, 166, 615

Wilson, R. E., \& Biermann, P. 1976, A\&A, 48, 349

Yi, S., Demarque, P., Kim, Y., et al. 2001, ApJS, 136, 417 